\title{
BMJ Open Study protocol for screening and diagnosis of fetal alcohol spectrum disorders (FASD) among young people sentenced to detention in Western Australia
}

\author{
Hayley M Passmore, ${ }^{1,2}$ Roslyn Giglia, ${ }^{1,3}$ Rochelle E Watkins, ${ }^{1,3}$ \\ Raewyn C Mutch, ${ }^{1,2,3,4}$ Rhonda Marriott, ${ }^{5}$ Carmela Pestell, ${ }^{1,6}$ \\ Stephen R Zubrick, ${ }^{1,3,7}$ Candice Rainsford, ${ }^{1}$ Noni Walker, ${ }^{1}$ James P Fitzpatrick, ${ }^{1,3}$ \\ Jacinta Freeman, ${ }^{1}$ Natalie Kippin, ${ }^{1}$ Bernadette Safe, ${ }^{1}$ Carol Bower ${ }^{1,3,8}$
}

To cite: Passmore HM, Giglia R, Watkins RE, et al. Study protocol for screening and diagnosis of fetal alcohol spectrum disorders (FASD) among young people sentenced to detention in Western Australia. BMJ Open 2016;6:e012184.

doi:10.1136/bmjopen-2016012184

- Prepublication history for this paper is available online. To view these files please visit the journal online (http://dx.doi.org/10.1136/ bmjopen-2016-012184)

Received 6 April 2016 Accepted 1 June 2016

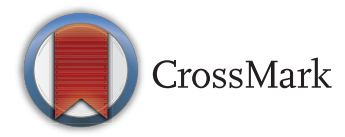

For numbered affiliations see end of article.

Correspondence to Hayley M Passmore; hayley. passmore@telethonkids.org. au

\section{ABSTRACT}

Introduction: Prenatal alcohol exposure can cause lifelong disability, including physical, cognitive and behavioural deficits, known as fetal alcohol spectrum disorders (FASD). Among individuals with FASD, engagement with justice services is common. Little is known about the prevalence of FASD among young people engaged with the Australian justice system. This study aims to establish FASD prevalence among sentenced young people in detention in Western Australia (WA), and use the findings to develop a screening tool for use among young people entering detention. Translation of these results will guide the management and support of young people in detention and will have significant implications on the lives of young people with FASD and the future of Australian youth justice services.

Methods and analysis: Any sentenced young person in WA aged 10-17 years 11 months is eligible to participate. Young people are assessed for FASD by a multidisciplinary team. Standardised assessment tools refined for the Australian context are used, acknowledging the language and social complexities involved. Australian diagnostic guidelines for FASD will be applied. Information is obtained from young people responsible adults, teachers and custodial officers. Individualised results and management plans for each young person are communicated to the young person and responsible adult. Prevalence of FASD will be reported and multivariate methods used to identify variables most predictive of FASD and to optimise the predictive value of screening.

Ethics and dissemination: Approvals have been granted by the WA Aboriginal Health Ethics Committee, University of WA Human Research Ethics Committee, Department of Corrective Services, and Department for Child Protection and Family Support. Anonymised findings will be disseminated through peer-reviewed manuscripts, presentations and the media. Extensive consultation with stakeholders (including government agencies, detention centre staff, community service

\section{Strengths and limitations of this study}

First study to estimate the prevalence of fetal alcohol spectrum disorder (FASD) among young people in detention in Australia.

- Aims to develop a screening tool for the identification of young people with FASD early in their involvement with the justice system.

- Incorporates research translation and is guided by extensive community consultation.

- Conducting research within a detention centre poses challenges and environmental instabilities.

providers, the young people and their families or carers) will be ongoing until findings are disseminated and translated.

\section{INTRODUCTION}

Prenatal alcohol exposure (PAE) can result in lifelong physical and neurocognitive abnormalities, which can severely impact on a person's quality of life. ${ }^{1-5}$ The spectrum of these abnormalities is termed fetal alcohol spectrum disorders (FASD). In Australia, the spectrum of FASD encompasses specific diagnoses depending of the severity and type of impairments present. $^{6}$ Diagnosis on the FASD spectrum include fetal alcohol syndrome (FAS), partial FAS (pFAS) and neurodevelopmental disorder-alcohol exposed (ND-AE). ${ }^{6}$ FAS is defined by evidence of growth deficiency, central nervous system (CNS) impairment in three or more domains, and the presence of all three specific facial features: short palpebral fissures, a smooth philtrum and a thin vermillion 
border of the upper lip. pFAS is defined by two of these three facial features and CNS impairment, with confirmed PAE. ND-AE is defined as CNS impairment across three or more domains without facial features, with confirmed PAE.

The primary disabilities seen in FASD can vary, but commonly include deficits in memory, learning, attention, understanding abstract concepts, reasoning, understanding cause and effect, learning from past experiences, information processing, decision-making and comprehending social skills or expectations. ${ }^{1-3} 57$ These deficits, a result of damage to the brain after PAE, can lead to a number of secondary effects that adversely impact an individual's well-being, productivity and life potential. These include school difficulties, unemployment, disrupted education, substance use and/or addiction, homelessness, mental health issues, and early and repeat engagement with the law. ${ }^{1} 7$

A large proportion $(60 \%)$ of young people with FASD become involved with the criminal justice system, ${ }^{1}$ and they are 19 times more likely to be detained compared with those without FASD. ${ }^{5}$ Young people with FASD often display hyperactivity, impulsiveness, aggressiveness and poor judgement, ${ }^{5} 89$ characteristics that can negatively impact on their ability to adhere to socially acceptable behaviours, hence becoming risk factors for offending. ${ }^{10}{ }^{11}$ In addition, young people with FASD often have an inability to understand causal relationships or remember that there are consequences for their actions. Although young people with FASD often become involved with the law due to their impulsive actions or aggressive behaviours, they can also be overly trusting of others, easily coerced or misled and victimised. $^{9}$

In addition to their increased risk of entering detention, young people with FASD have a high risk of exhibiting antisocial or undesirable behaviours while incarcerated. ${ }^{9}$ Justice, law enforcement and correctional staff might interpret these behaviours as intentional misconduct, rather than recognising that the behaviours are a result of neurocognitive impairment, interrupted education and possible comorbid mental health issues. Young people with FASD also have difficulty complying with parole orders that comprise stringent directives (eg, meeting in an unfamiliar place, adhering to a specified time), resulting in breaches of parole conditions and a potential return to incarceration. ${ }^{9} 12$ It is essential that FASD is identified as early as possible in a detained young person's contact with the law in order for their behaviours to be interpreted correctly as a manifestation of their impairments, and provide opportunity for appropriate application of the law and evidence-based management strategies throughout their engagement with justice services.

No prevalence rates for FASD among young people in Australian detention centres have been established. However, past research leads to the expectation that many cases of FASD are undiagnosed or have unintentionally been misdiagnosed as another disorder. ${ }^{5}{ }^{13-15}$ What has been documented is higher rates of FASD among Aboriginal people in Australia in comparison to non-Aboriginal people. ${ }^{16-18}$ Additionally, Indigenous ${ }^{\mathrm{i}}$ young people are 31 times more likely to be in detention compared with non-Indigenous young people across Australia. ${ }^{19}$ With those considerations in mind, it is anticipated that there will be many young people within detention in Western Australian who are affected by FASD.

In order to reduce the negative impact of crime and incarceration on society, there is a critical need to determine the extent that young people with FASD are involved with the justice system. ${ }^{20} 21$ Once that determination is made, development of appropriate evidencebased management strategies for these young people can take place. Screening for and diagnosis of FASD can be challenging for a number of reasons. There are often multiple barriers impeding diagnosis. ${ }^{13}$ Barriers can consist of minimal awareness among health professionals regarding PAE, difficulties in obtaining confirmation of alcohol use during pregnancy and the stigma often associated with diagnosis. ${ }^{22} 23$ Effective screening tools are essential for early identification of impairment. ${ }^{15} 24$ Unfortunately, there is a lack of screening instruments for FASD that are valid and reliable within an Australian context. ${ }^{25}$ Further complicating the issue is limited capacity for screening and diagnosis due to geographical, service funding and training constraints. ${ }^{22} 2627$

Ideally, the diagnostic process involves a comprehensive assessment of a young person's health, neurocognition, sensory, motor, speech and social skills carried out by a multidisciplinary diagnostic team. ${ }^{28}$ Information should be obtained from those close to the individual such as family members and teachers, and external sources including school records, family history, medical records, social services information, and in particular, any previous diagnoses of physical or cognitive impairment to verify what is reported by the individual. ${ }^{28}$ It is crucial that the diagnostic process is tailored to the needs of the individual, and that the information ascertained is communicated back to the individual, their family and other supports as appropriate. By identifying the individual's strengths and difficulties in this way, their social, health and educational outcomes can be considerably improved, and the risk of secondary effects decreased with the use of appropriate management strategies and modification of their physical and social environments. ${ }^{29}$

With no indications as to how many people in the Australian justice system are living with FASD, it is impossible to ensure the required supports are available for

${ }^{\mathrm{i}}$ The term Indigenous has been used to refer to Aboriginal and Torres Strait Islander people. The term Aboriginal has been used elsewhere to refer to Aboriginal people only, given the specific involvement of Aboriginal young people in this research. 
those affected. This is likely to result in a large proportion of individuals with physical and cognitive impairments maintaining their involvement with the law due to justice systems not having the skills, resources or support services required to break the cycle of recidivism. ${ }^{30}$ Awareness of these issues in Western Australia (WA) is growing, with recent parliamentary enquiries, ${ }^{31-33}$ community services providers ${ }^{34}$ and the WA Department of Health FASD Model of Care ${ }^{35}$ calling for programmes to identify and assist young people with FASD while in detention to help them function better when out in society, and help prevent them from returning to detention.

In response, this study was undertaken to determine the extent that FASD exists among young people in detention in WA, and guide the youth justice system in the identification, management and support of those affected. The study is funded by a National Health and Medical Research Council (NHMRC) Targeted Research Grant for Fetal Alcohol Spectrum Disorders in Indigenous Populations. This protocol paper describes the consultations that guide the study processes, and the methods and assessment tools selected and pilot-tested prior to beginning the study.

\section{Study aims}

The aims of this research are to:

1. Estimate the prevalence of FASD among young people in detention in WA.

2. Develop and evaluate a FASD screening instrument appropriate for use among young people entering detention in Australia.

\section{METHODS}

While this paper outlines the screening and diagnosis protocols used in the prevalence study, it is important to note that this is only one component of a much larger study. This overarching study is multifaceted and incorporates research translation, empowerment and capacity building to ensure diagnosis is not carried out in isolation, and provides opportunity for positive change to occur in the lives of those affected by FASD. More specifically, additional research components (as shown in figure 1) involve building capacity among the detention centre workforce, qualitative evaluation of the prevalence study processes and an analysis of linked data once the prevalence study is completed to explore risk factors for involvement with the justice system.

\section{Setting}

The study is being conducted in Banksia Hill Detention Centre, the only detention centre within the state of WA for young people who offend. It was built in 1997 and is located $\sim 26 \mathrm{~km}$ from the Perth city centre. Since January 2013, the detention centre population has fluctuated between 110 and 207 young people, but is currently at 142 young people at the time of publication. The population consists of young people on remand, those awaiting trial or bail determination and those who have been sentenced to detention. Males and females reside in separate residential units within the facility, and $\sim 75 \%$ of the population at present are Aboriginal. ${ }^{36}$

\section{Ethics and dissemination}

Given the characteristics of the population under investigation and the seriousness of the potential diagnostic outcomes, cultural sensitivity is of primary concern.

Anonymised results of this study will be widely disseminated through peer-reviewed manuscripts, conference presentations, the media and consultations with relevant stakeholders, government agencies, justice professionals and community organisations where appropriate. No personally identifiable information will be released without assent from the participating young person and consent from their responsible adult.

Figure 1 Design of overarching study.

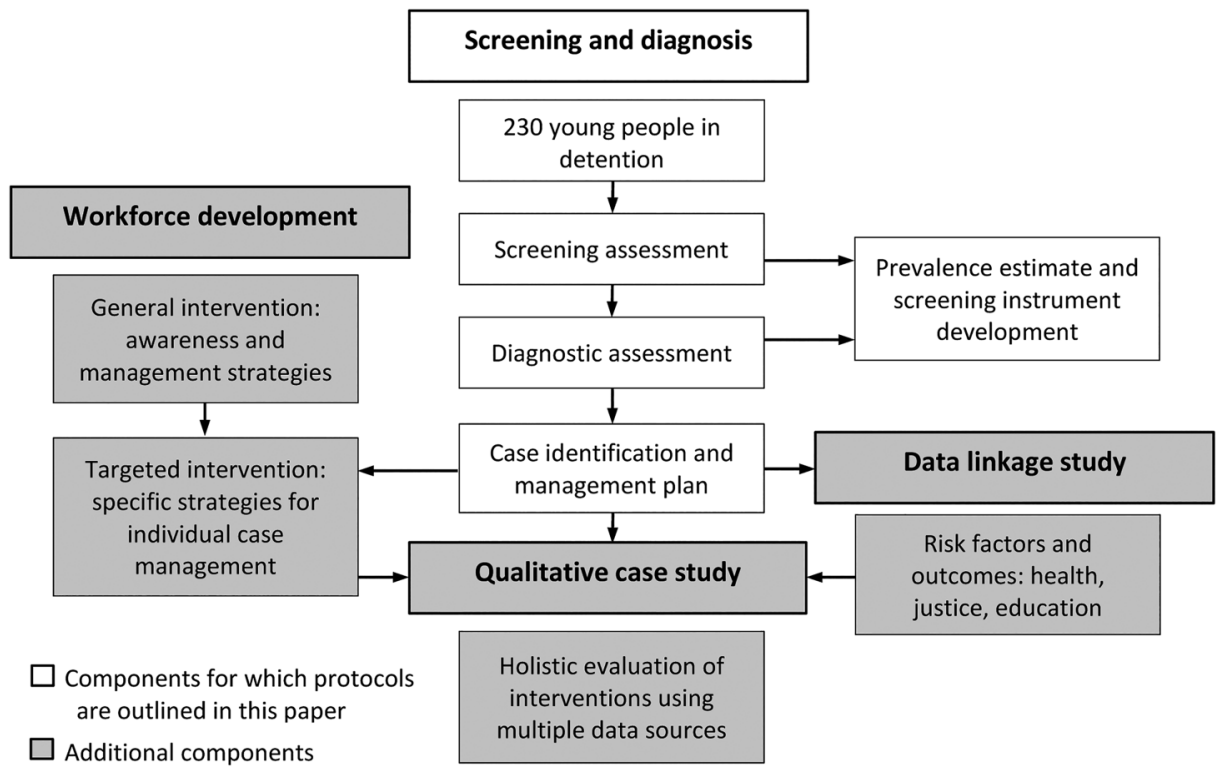




\section{Consultation}

Prior to the grant submission, extensive consultation with key stakeholders was undertaken over several months to determine if the research would be feasible and acceptable to the Department of Corrective Services (DCS) and the Indigenous and non-Indigenous communities affected by the research. With initial high-level support for the study, consultations were held once the grant was approved and prior to the start of a pilot test of assessment processes involving the clinical assessment of 10 young people. A significant amount of time was spent developing networks and working relationships for these multilayered consultations to occur, such as with staff from youth justice services, child protection services, Aboriginal health networks, FASD advocates and academic institutions. These consultations are ongoing, and are essential to ensure adequate departmental and community involvement in the research and its practical application. Many of these consultations have been facilitated by the development of three specific groups created to inform the research: a Consumer and Community Reference Group, a Steering Group and a DCS and Department for Child Protection and Family Support (CPFS) Reference Group.

Comprised of foster parents with a child with FASD, an Indigenous researcher investigating the impact of FASD in Aboriginal families, and an Indigenous community worker who has also fostered children with FASD, the Consumer and Community Reference Group is a cornerstone for the study. It was integral to the development of the recruitment resources such as the pictorial assent forms, the consent forms and the information provided to participants and their responsible adult. This group also provided advice on: (1) culturally appropriate communication strategies for engaging staff families and the young people, as well as for processes of obtaining consent; (2) best practice when asking for sensitive information; (3) the possibility of reimbursements for the young people; (4) the dissemination of information and (5) potential challenges. In addition, a community navigator who had previously been employed as a youth custodial officer at the detention centre was employed prior to the pilot test to provide assistance in developing the recruitment information and in navigating the operational aspects of the detention centre system and DCS protocols. Other community members and community organisations are contacted for comment and guidance as required, on recommendations from the Consumer and Community Reference Group.

Representatives from relevant consumer and advocacy organisations, the Aboriginal Legal Service, the Office of the Inspector of Custodial Services, community members and a senior administrative staff member from the detention centre comprise the Steering Group. The group meets bi-monthly and provides direction and guidance on the research processes and how best to draw on the research outcomes to positively influence change in the justice setting, while maintaining the wellbeing of the young people as their primary interest.
The Reference Group is a high-level directorial group that addresses the ongoing challenges of conducting research in a custodial setting. Decision makers from within the DCS, CPFS and detention centre meet to ensure that research protocols and/or operational procedures can be adjusted to meet the requirements of the detention environment while still maintaining the rigour of the research methodology. The Deputy Commissioner for Youth Justice Services, DCS senior managers from health services and psychology, and staff from the detention centre including the superintendent, senior administrators, an Aboriginal welfare officer, a senior representative from CPFS, chief investigators of the study and the project manager comprise the membership of this group.

All three groups have been integral in the development of the protocols, and they continue to provide guidance across many other aspects of the study. Since beginning the pilot test, several changes have taken place following consultations with those most appropriate for each particular issue. For example, consultations with the Reference Group have resulted in the detention centre providing a physical space more suitable for clinical assessments than was initially used, and the supervision procedures of detention centre staff were altered to ensure staff time is used most effectively by reducing additional staff allocations while still maintaining appropriate levels of supervision during assessments.

Aside from these groups, information about the study processes has and will continue to be widely communicated as appropriate. Formal presentations have been held for all departments at the detention centre including case management, psychology, administration, education, youth custodial officers and youth justice officers, as well as for a number of community organisations deemed relevant to the study outcomes. Opportunistic meetings, discussions and 'yarning' continue to provide valuable input into shaping the research processes. Informal conversations with parents and guardians of the young people, community workers and detention centre staff occur as opportunities arise. The 'elevator pitch' has quickly become the 'carpark conversation' as many exchanges occur in passing, either inside the detention centre or outside in the detention centre carpark.

While considerable time and resources have been spent on these formal and informal consultations, they continue to provide invaluable insight and collaborative opportunities that shape not only the scope and processes of the study, but also its impact on the lives of young people affected by FASD.

\section{Participants}

All Aboriginal and non-Aboriginal young people who are sentenced to a period of detention within the centre and are aged $10-17$ years 11 months are eligible to participate. 


\section{Recruitment}

Participants are recruited by a direct (face-to-face) approach made initially by a research officer based at the detention centre, with support from custodial officers responsible for supervision of the young people, detention centre teaching staff, case managers and other support staff. If a young person expresses interest in being involved in the study, then the research officer will explain the purpose of the study using pictorial assent forms and participant information sheets which the young person can retain.

Young people who are serving a sentence of detention and have consequently been deprived of certain civil liberties have a vulnerable status with respect to their ability to consent for research. In this study, a young person is the first to provide written assent to participate in the study. With their assent, a study information sheet is then given to the identified responsible adult from whom written consent is obtained. Consent for young people in the care of CPFS is sought directly from the CPFS case manager responsible for that young person. Once permission from CPFS has been granted, parents of a young person in the care of CPFS will be contacted by the research officer for consent as a courtesy and information sharing opportunity. In some cases, CPFS may determine that it is not appropriate for the research officer to contact the parents of some children in their care, and no attempt will be made to contact these parents. The attainment of assent and consent will set into motion the data collection processes of the research study.

\section{Data collection processes}

The research officer is responsible for the collection of information using standardised forms from the participant, the responsible adult, detention centre teachers, youth custodial officers and child protection case workers. They are also responsible for taking photos of the young person's face and entering them into Astley's FAS Facial Photographic Analysis software package (FAS Facial Photographic Analysis Software [program]. 2.0 version. Seattle, WA: University of Washington, 2012.), a program designed to effectively measure and rank the extent that the facial features specific to FAS and pFAS are present. Table 1 outlines all information collected and methods of collection used.

The research officer schedules the clinical assessments for the participants. Assessments for each individual are conducted within a period of 2 weeks depending on the operational requirements of the detention centre, availability of custodial officers who must accompany the young person to the assessment, clinician availability and the requests of the young person themselves. If necessary, any of the assessments can be offered in several smaller sessions if the young person is unable to complete them in one sitting. Most assessments are scheduled in the mornings to reduce chances of fatigue in the afternoon.

\section{Diagnostic criteria}

In order to make a correct diagnosis of FASD, the multidisciplinary team must conduct a full assessment of the young person's facial features, growth and CNS function. In addition, a diagnosis also requires information on PAE. The Australian guidelines for the diagnosis of FASD recommended by Watkins $e t a l^{6}$ are used in this study to assign specific diagnoses on the spectrum of FASD based on the comprehensive diagnostic assessment by the study clinicians and information collected. Table 2 outlines the recommended Australian categories and criteria for diagnosis.

\section{Diagnostic assessment}

The diagnostic assessment is conducted by a team comprised of a paediatrician, neuropsychologist, speech pathologist and occupational therapist. Table 3 outlines all of the formal and informal assessments used.

The paediatrician completes a comprehensive health assessment, developed following a literature review and consultation with national and international colleagues experienced in the care of children and young people with FASD and within justice services. The paediatrician conducts an interview with the young person, deriving their early life, educational history and age of onset for health risk behaviours. In addition, the young person participates by drawing their family tree, and in this activity discloses their lived history of nurture and trauma. Clinical history of current and past health issues is obtained using standardised health history questionnaires ${ }^{4647}$ including a Western Australian-derived structured interview for cross-cultural paediatric healthcare. ${ }^{45}$ Clinical examination assesses the young person's mood and wellness; social strengths; expressive abilities; growth; visual acuity and hearing; fine and gross motor function; dysmorphic features; neurocutaneous stigmata; and cardiovascular, respiratory and neurological function. Evidence of previous injuries and any other abnormal physical findings are documented in a body map, including poorly healed fractures, lacerations, tattoos and piercings. Measures of growth (head circumference, weight and height), palpebral fissure lengths, lip philtrum shape and upper lip volume are recorded, and additional features associated with PAE are systematically noted, including hair whorl, clinodactyly and shape of palmar creases.

The assessment conducted by the neuropsychologist was developed following a comprehensive literature review, and in consultation with experienced clinical neuropsychologists familiar with conducting assessments within community and youth justice settings. The formal neuropsychology assessment battery was chosen to adequately cover the domains used to determine whether there is functional CNS dysfunction (ie, 2 SD below the mean) in three or more domains. As per Australian diagnostic criteria, ${ }^{6}{ }^{63}$ the domains assessed are intellectual functioning/cognition, attention/activity levels/sensory, executive functions, memory, social skills/adaptive 
Table 1 Data collected and method of collection

\begin{tabular}{ll}
\hline Person contacted & Assessment/survey \\
\hline Participant & $\begin{array}{l}\text { Assent form } \\
\text { Facial photos }\end{array}$ \\
& \\
Responsible adult & $\begin{array}{l}\text { Biomedical and psychosocial checklist } \\
\text { Consent }\end{array}$
\end{tabular}

Background history survey (includes AUDIT-C $C^{37}$ questions for prenatal alcohol use) CBCL Parent's Report Form ${ }^{38}$

Vineland Adaptive Behaviour Scales Second Edition-parent/caregiver rated form ${ }^{39}$

$(\text { BRIEF-P) })^{40}$

\begin{tabular}{|c|c|}
\hline \multirow[t]{2}{*}{ Teacher } & $\begin{array}{l}\text { Vineland Adaptive Behaviour Scales, } \\
\text { Second Edition-teacher rated form }{ }^{41 *} \\
\text { BRIEF- } T^{40 *}\end{array}$ \\
\hline & CBCL Teacher's Report Form ${ }^{38 *}$ \\
\hline $\begin{array}{l}\text { Youth custodial } \\
\text { officer }\end{array}$ & $\begin{array}{l}\text { Informant Brief Screener (adapted from } \\
\text { Conry and Asante) }{ }^{42}\end{array}$ \\
\hline & $\begin{array}{l}\text { Life Skills Checklist (adapted) }{ }^{43} \dagger \\
\text { Informal social skills and social } \\
\text { communication questionnaire }\end{array}$ \\
\hline Child protection & Background history survey as above ${ }^{*}$ \\
\hline
\end{tabular}

\section{Method of collection}

Collected in person by research officer

Taken by research officer and entered into photo analysis software program (FAS Facial Photographic Analysis Software [program]. 2.0 version. Seattle, WA: University of Washington, 2012)

Collected in person by research officer

Collected either in person by the research officer, in person by a youth justice officer for families in regional and remote towns, or via post, via email or via fax after communication with the research officer has occurred in person or via telephone

By research officer either in person or via telephone

Distributed in person by research officer, self-completed by teachers and returned to research officer in person

Distributed in person by research officer, self-completed by youth custodial officer and returned to research officer in person

Retrieved from case notes of young people in care of CPFS by case worker and given to research officer

${ }^{*}$ Only if responsible adult not available to complete.

†Only if identified as Aboriginal or Torres Strait Islander.

BRIEF-P, Behaviour Rating Inventory of Executive Function-Parent; BRIEF-T, Behaviour Rating Inventory of Executive Function-Teacher; CBCL, Child Behaviour Checklist; CPFS, Child Protection and Family Support.

functioning/social communication and academic functioning. The domains of language and motor ability are more thoroughly assessed by the speech pathologist and occupational therapist on the team. Additional considerations for the neuropsychology tests (and questionnaires) chosen included their psychometric properties (reliability and validity), administration time, clinical utility (including use of engaging culturally appropriate materials) and whether Australian norms were available. A non-verbal test of intelligence was also chosen for those without standard Australian English, and a Life Skills Checklist was developed for Aboriginal children as a more culturally appropriate method of assessing adaptive functioning. No changes were made to the neuropsychology assessment battery following the pilot test.

Speech, language and communication are assessed by a speech pathologist. Development of the assessment battery included a comprehensive literature review and liaison with clinicians and research academics in the fields of FASD, youth justice, language learning impairments and language assessment of people who speak Australian Aboriginal English and Australian Aboriginal languages. Standardised assessments were chosen for their reliability and validity in measuring receptive and expressive oral language, and phonological awareness of young people in the target group age range ${ }^{53} 55$ Oral language and phonological awareness are included in the Canadian guidelines for FASD diagnosis, ${ }^{28}$ and standardised assessments have been used widely in examining oral language profiles of children with FASD and young offenders. ${ }^{64-67}$

It is recognised that standardised norm referenced assessments are based on monolingual standard Australian English language acquisition. For children who are learning standard Australian English as a second dialect or language, it is possible that their language differences may be misinterpreted for language disorder, causing bias and invalid results. ${ }^{68-70}$ Also, a child's health, social background, schooling consistency, culture and parent/caregiver literacy levels are just some examples of factors possibly affecting progression to a competent level of standard Australian English. ${ }^{71}$ Therefore, informal tasks have been developed to assist in examining the language abilities of the young people 
Table 2 Watkins et a ${ }^{\hat{\beta}}$ recommendations for Australian FASD diagnostic categories and criteria

\section{Diagnostic category}

\section{Diagnostic criteria*}

FAS

Requirements for Requires all 4 of the following criteria

diagnosis to be met:

\section{Confirmed or unknown}

Facial anomalies Presence of all 3 of the following facial anomalies at any age:

1. Short palpebral fissure length (2 or more SDs below the mean)

2. Smooth philtrum (rank 4 or 5 on the UW Lip-Philtrum Guide ${ }^{44}$ )

3. Thin upper lip (rank 4 or 5 on the UW Lip-Philtrum Guide ${ }^{44}$ )

Growth deficit Prenatal or postnatal growth deficit indicated by birth length or weight $\leq 10$ th centile adjusted for gestational age, or postnatal height or weight $\leq 10$ th centile

CNS abnormality At least 1 of the following:

1. Clinically significant structural abnormality (eg, occipital-frontal circumference $\leq 3 \mathrm{rd}$ centile, abnormal brain structure), or neurological abnormality (seizure disorder or hard neurological signs); and/or

2. Severe dysfunction (impairment in 3 or more domains of function, 2 or more SDs below the mean)§

†Based on the presence of two of the three characteristic FAS facial features, the observed impairments cannot be causally linked to PAE. $\ddagger$ Not required for diagnosis but may be present.

$\S A s s e s s m e n t$ of dysfunction based on evidence from standard validated assessments instruments interpreted by qualified professionals.

CNS, central nervous system; FAS, fetal alcohol syndrome; FASD, fetal alcohol spectrum disorder; ND-AE, neurodevelopmental

disorder-alcohol exposed; PAE, prenatal alcohol exposure; pFAS, partial FAS; UW, University of Washington.

who speak Australian Aboriginal English or an Aboriginal language as their first or 'best' language. These tasks were trialled and revised during the pilot test, and are administered by the speech pathologist with an interpreter from the Aboriginal Kimberley Interpreting Service. In addition, informal assessments and checklists were developed to examine motor speech, connected discourse (narrative), and social communication and social skills. These areas of speech, language and communication are included in the Canadian guidelines ${ }^{28}$ and were used by Salter $^{72}$ in a FASD prevalence study in WA's Fitzroy Valley. ${ }^{73}$ It was planned that higher level language would be measured using the Clinical Evaluation of Language Fundamentals, Fifth Edition, Metalinguistic $;{ }^{74}$ however, due to time constraints, this was removed after the pilot test.

The assessment of motor skills and sensory processing is conducted by the occupational therapist. The development of the assessment process was underpinned by an investigation into the current Australian and international literature and in consultation with other therapists working in the FASD research field. The Quick Neurological Screening Test-Third Edition ${ }^{60}$ is used to review any underlying soft neurological signs such as visual tracking, motor planning, coordination and balance. The Movement Assessment Battery for Children-Second Edition (Movement ABC-2) was chosen to assess fine and gross motor coordination as it is quick assessment to administer and score, with strong validity and reliability. ${ }^{59}$ However, use of the Movement ABC-2 is limited in that normative scores are only available for those aged between 3 and 16 years. Alternative assessments that cover a greater range of ages were considered for use but deemed too lengthy for the study schedule, and it was decided that the Movement ABC-2 would be maintained as a limitation when used in the study with young people over 16 years old. The Beery Visual Motor Integration assessment and subtests (Visual Perception and Motor Coordination) were chosen due to their high reliability and validity, ${ }^{58}$ and findings of past research which shows a correlation between visual motor integration skills and a FASD diagnosis. ${ }^{75}$ The Sensory Profile-Adolescent/Adult self-questionnaire was used, as it is the only tool that covers the required age range, and has been used widely in other research investigating sensory processing difficulties contributing to attention and activity levels. ${ }^{61}$ An informal handwriting sample was also completed to review any existing handwriting difficulties. All motor and sensory 
Table 3 Diagnostic assessments used by multidisciplinary diagnostic team

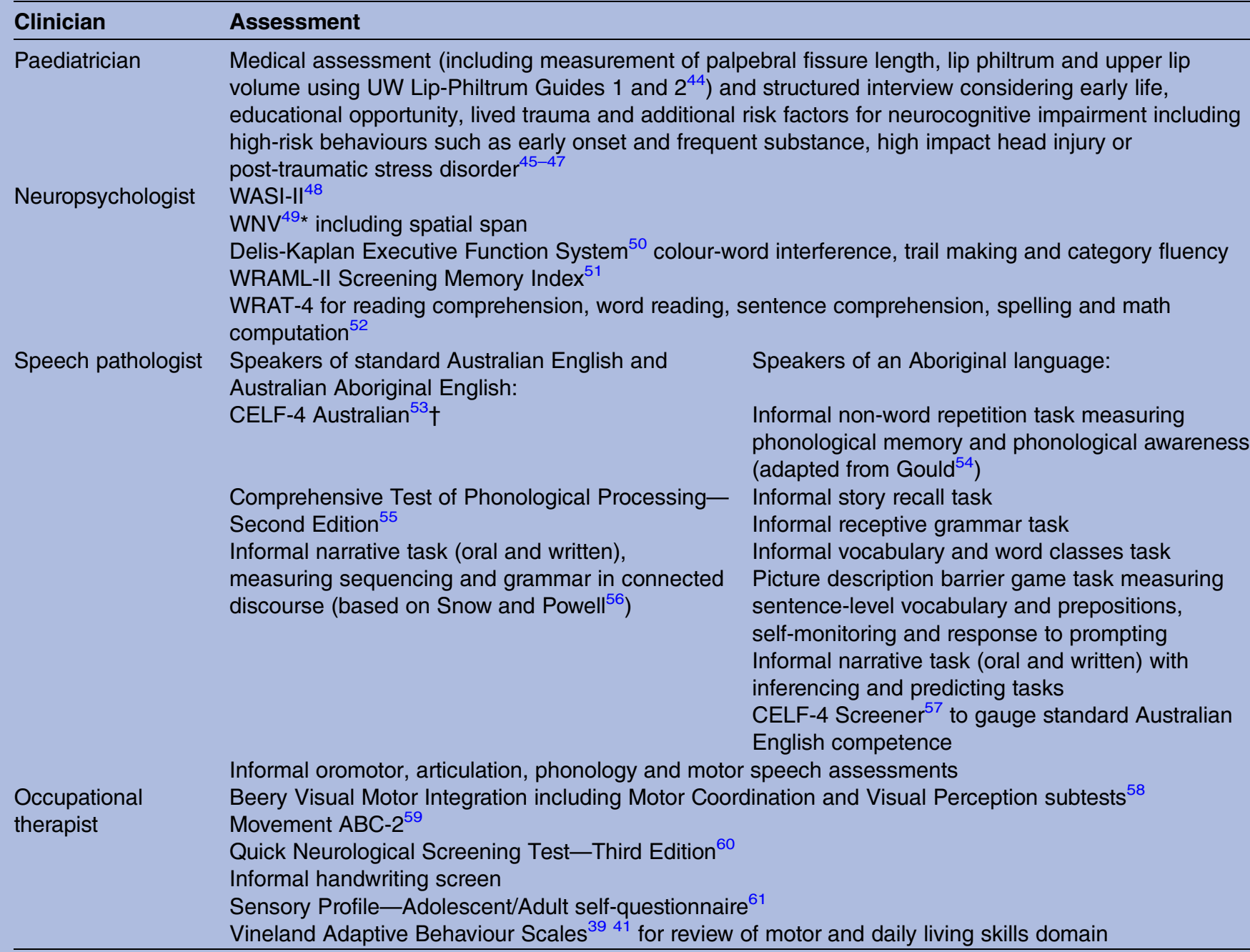

*WNV administered instead of the WASI if participant cannot speak fluent Australian English.

†For speakers of Australian Aboriginal English responses were coded in standard Australian English, and with aspects of Australian Aboriginal English grammar based on literature including Pearce and Williams. ${ }^{62}$

CELF-4 Australian, Clinical Evaluation of Language Fundamentals, Fourth Edition, Australian Standardised Edition; CELF-4 Screener, Clinical Evaluation of Language Fundamentals, Fourth Edition, Screening Test Australian \& New Zealand Language Adapted Edition; Movement ABC-2, Movement Assessment Battery for Children-Second Edition; UW, University of Washington; WASI-II, Wechsler Abbreviated Scale of Intelligence-Second Edition; WNV, Wechsler Non-Verbal Test of Intelligence; WRAML-II, Wide Range Achievement in Memory \& LearningSecond Edition; WRAT-4, Wide Range Achievement Test-Fourth Edition.

processing assessments were trialled during the pilot test, and deemed to be the most appropriate tools for this study. No changes were made following the pilot test; however, a supporting resource was developed to aid the young people when completing the Sensory Profile questionnaire.

\section{Data collation}

Once all clinical assessments are complete and all available questionnaire information has been collected for an individual, the multidisciplinary team meets to review the results and make a diagnosis if appropriate, taking into account co-occurring morbidities such as attentiondeficit/hyperactivity disorder or intellectual disability. Together, the team prepares an 'Assessment Results and Recommendations Report' in a format appropriate for the participant and their responsible adult, building on the young person's strengths and providing individualised management recommendations. For example, specific communication styles and behaviour management strategies to best support the young person while in detention and on release will be outlined. The report is the property of the participant and their responsible adult, but with their permission it can be released to detention centre staff, medical and mental health professionals, youth justice services and other agencies as necessary.

On completion of the report, a member of the multidisciplinary team meets with the young person and their responsible adult individually, to communicate the assessment results and recommendations in a manner most appropriate for their level of comprehension. This 
provides an opportunity for the participants and their responsible adults to ask questions or raise concerns regarding the assessment process and outcomes. Support is offered during these feedback sessions in the form of a trusted adult present on request of the young person, or a translator for those with standard Australian English as their second language. Contact details are provided should the responsible adult want additional information or support, and young people are reminded of the support available at the detention centre, including health and psychology services.

\section{Screening instrument}

A key component of the study is the development and evaluation of a screening instrument that can be used to screen all young people entering detention to identify risk factors for FASD. The screening instrument will be developed after completion of the data collection, based on potential screening risk factors and the diagnosis of FASD in the study sample.

\section{Statistical considerations}

Sample size estimation was based on the midpoint estimate of the proportion of young people from custodial correctional populations with FASD (15\%) in a recent systematic review. ${ }^{5}$ Assuming a CI width of $10 \%$, and an $\alpha$ of 0.05 , the number of participants required to establish that the screening test is at least $80 \%(90 \pm 10 \%)$ sensitive is 230. The study is powered on the number of participants required to ensure acceptable precision of the sensitivity estimate as fewer participants are required to establish precise estimates of specificity. ${ }^{76}$

Multivariate methods including multiple logistic regression modelling will be used to identify the screening indicators most predictive of a diagnosis of FASD and which optimise the predictive value of screening. Receiver operating characteristics curve analysis will be used to facilitate selection of optimal screening cut-points to examine trade-offs in sensitivity versus specificity and balance the costs of over-referral and underascertainment in screening instrument development.

\section{DISCUSSION}

This study is the culmination of over three decades of research work in the area of FASD. ${ }^{6}{ }^{16} 77-81$ It will be the first to determine prevalence rates of FASD among young people in an Australian juvenile detention centre. Results of this research will be used to develop appropriate interventions and support for young people diagnosed with FASD within the justice system. It is likely, based on previous research, ${ }^{15} 138283$ that many of the adversities exhibited by young people within detention may be attributed to FASD and that a lack of diagnosis in the Australian context has led to suboptimal targeting of behavioural interventions within the justice system. This research will provide an opportunity to build on the clinical practice models for improving treatment effectiveness ${ }^{24}$ by providing diagnoses or other clinical findings alongside a tailored report that includes individual recommendations for the benefit of each young person.

Conducting research within a juvenile detention centre poses challenges with environmental limitations or instabilities expected to arise as the study progresses. These challenges could involve the space available within the detention centre for the assessments to take place, the availability of officers to escort the young people to the assessments and access to the young people if they have been involved in an incident or do not feel like participating on a particular day. Owing to such challenges, flexibility in study design will remain a necessity, without compromising the integrity of the research. Since moving from the pilot test to the definitive study, research processes have been modified according to operational requirements of the detention centre and the various complexities existing within participants' lives. Such alterations include (1) the adaption of the speech, language and communication assessment battery to accommodate for time constraints; (2) changing the physical areas of the detention centre used for assessments; (3) altering reporting guidelines in the case of being privy to information or an incident that requires reporting under detention centre policy and (4) changing the process of providing feedback to the young people and their responsible adults. While challenges are unavoidable due to the complex nature of the detention centre environment, ongoing consultation with stakeholders provides opportunity to find solutions that appropriately balance operational procedures with research integrity and study quality.

Widespread consultations have also led to the multifaceted design of the overarching study, which will have major implications on the significance of the research and its translation. While the prevalence study encompasses diagnosis within a small population of high-risk young people, findings will be translated into a validated screening tool that can be used to identify young people living with an undiagnosed FASD early in their contact with the justice system. The ability to screen young people and refer them for clinical FASD assessment enables a pathway of management to be determined for the young person, their families and their carers, ${ }^{35}$ including corrective services. However, it is the workforce development and qualitative evaluation components that will play a crucial role in the translation of the research, and facilitate change within the Australian youth justice system.

The workforce development component will occur simultaneously with the prevalence study. It involves the implementation and evaluation of an intervention aiming to increase knowledge and awareness of FASD among custodial staff, and build their capacity to manage young people suspected to have FASD and other cognitive impairments using equitable and culturally appropriate management strategies. The intervention will be developed in consultation with custodial staff 
members and using preliminary results from the prevalence study, to ensure that it is relevant to the staff and young people at the detention centre. Building the capacity of the detention centre workforce by empowering them with the skills to recognise and manage the behaviours of young people with neurodevelopmental disorders such as FASD will be a primary outcome of this planned work and a turning point for detention centres across Australia. Similar professional development and workforce education has been carried out with other professionals in the Western Australian justice sector, including the recent production of videos aiming to educate lawyers and magistrates about the relevance of FASD in their arenas of work. ${ }^{84}$

The qualitative evaluation will involve techniques suited to the complex identification and management of FASD within the justice context ${ }^{85}$ and aims to evaluate the impact of this research on the lives of the young people, their families and their wider communities, including the government agencies and community service providers engaging with the young people. This component will explore the successes and failures of the study, and is essential to understand if the screening and diagnosis and workforce development interventions facilitate cultural change across the youth justice system. The recognition that this research will not only affect the individual participants but the wider social context in which they live has been imperative throughout the study development and community consultations, and the qualitative evaluation component enables this to be further explored.

Together, the components of this research, including the linked data analysis, will crucially broaden the understanding of FASD and the youth justice system in Australia, while taking a humanitarian approach to research and research translation. Given that young people with FASD are at great risk of obtaining and maintaining involvement with the justice system, it is crucial that research in this area is translated into meaningful and enduring outcomes, ultimately improving the lives of young people with FASD who are engaged with the justice system Australia wide.

\footnotetext{
Author affiliations

${ }^{1}$ Telethon Kids Institute, The University of Western Australia, Perth, Western Australia, Australia

${ }^{2}$ School of Paediatrics and Child Health, The University of Western Australia, Perth, Western Australia, Australia

${ }^{3}$ National Health and Medical Research Council Centre of Research Excellence "Reducing the Effects of Antenatal Alcohol on Child Health", Perth, Western Australia, Australia

${ }^{4}$ Department of Health Western Australia, Child and Adolescent Health Service, Perth, Australia

${ }^{5}$ School of Psychology and Exercise Science, Murdoch University, Perth, Western Australia, Australia

${ }^{6}$ School of Psychology, The University of Western Australia, Perth, Western Australia, Australia

${ }^{7}$ Faculty of Education, The University of Western Australia, Perth, Western Australia, Australia

${ }^{8}$ Centre for Child Health Research, The University of Western Australia, Perth, Western Australia, Australia
}

Acknowledgements The authors thank all of the young people at Banksia Hill Detention Centre involved in the study and their families for their participation and support. They also thank all members of the Consumer and Community Reference Group, the Steering Group and the Reference Group for their invaluable and continuing input to the study. They thank the Department of Corrective Services and the Department for Child Protection and Family Support for their ongoing support, and acknowledge all of their staff members involved with the study including all staff based at Banksia Hill Detention Centre. The authors acknowledge Professor Jonathan Carapetis, for his ongoing input and support as an associate investigator on the study. They also thank individuals who have contributed to the study: Scott Avery, Peter Collins, Steven Dhu, Aaron Dick, Damian Griffis, Heather Jones, Glenn Pearson, Neil Reynolds, Vicki Russell, Julie Waud and Robyn Williams. This work was supported by the NHMRC Reducing the Effects of Antenatal Alcohol on Child Health Centre of Research Excellence (\#1110341).

Contributors $\mathrm{CB}, \mathrm{REW}, \mathrm{RCM}$ and RM are chief investigators on the study, and JPF, CP and SRZ are associate investigators. HMP is a PhD student with The University of Western Australia. HMP and RG wrote the first draft of the manuscript, and HMP was responsible for compiling the final draft. RG was the researcher responsible for overseeing the manuscript and the implementation of the pilot test. REW was the primary researcher responsible for the concept development, research design and grant application. RCM is a paediatrician and researcher and was integral to the development of the concept, clinical assessment battery and study processes. RCM and CP, along with NK and BS, comprise the clinical team for the study who were each responsible for developing the assessment battery relevant to their field, and drafting parts of the manuscript relating to the clinical assessments. RM is a researcher specialising in Aboriginal health and well-being, and was involved in the grant application and concept development. JPF is a paediatrician and researcher who provided input into the concept development and clinical assessment battery. SRZ is a chief investigator in the Australian Research Council Centre of Excellence for Children and Families over the Lifecourse (CE140100027) and provided input into the concept development and grant application. CR was the project officer for the research at the time of pilot implementation and integral to operationalising the research design, project databases and gaining ethical approval. NW is the project manager and is responsible for managing the governance and community consultations relevant to the research, developing study protocols and gaining ethical approval. JPF, as the research officer based at the detention centre, is responsible for consumer and community consultations, recruitment processes and coordination of clinical assessments. CB is the senior researcher responsible for overseeing the grant application and research design, and continues to oversee the reliability and protocols of the study. All authors read, edited and approved the final manuscript.

Funding This research is funded by the National Health and Medical Research Council (grant number APP1072072). CB was funded by a National Health and Medical Research Council Research Fellowship (\#634341). HMP is funded by an Australian Postgraduate Award Scholarship and The University of Western Australia Safety Net Top-up Scholarship (reference number 21806348)

\section{Competing interests None declared}

Ethics approval Ethics approval has been granted by the Western Australian Aboriginal Health Ethics Committee (approval number 582) and University of Western Australia Human Research Ethics Committee (approval number RA/4/ 1/7116). Research approvals were also gained from the Department of Corrective Services (project ID 335) and the Department for Child Protection and Family Support (approval number 2015/8981)

\section{Provenance and peer review Not commissioned; externally peer reviewed.}

Open Access This is an Open Access article distributed in accordance with the Creative Commons Attribution Non Commercial (CC BY-NC 4.0) license, which permits others to distribute, remix, adapt, build upon this work noncommercially, and license their derivative works on different terms, provided the original work is properly cited and the use is non-commercial. See: http:// creativecommons.org/licenses/by-nc/4.0/ 


\section{REFERENCES}

1. Streissguth AP, Bookstein FL, Barr HM, et al. Risk factors for adverse life outcomes in fetal alcohol syndrome and fetal alcohol effects. J Dev Behav Pediatr 2004;25:228-38.

2. Stratton K, Howe C, Battaglia F. Fetal alcohol syndrome: diagnosis, epidemiology, prevention, and treatment. Washington, WA: Institute of Medicine and National Academy Press, 1996.

3. Koren G, Nulman I, Chudley AE, et al. Fetal alcohol spectrum disorder. CMAJ 2003;169:1181-5.

4. Clark E, Lutke J, Minnes $P$, et al. Secondary disabilities among adults with fetal alcohol spectrum disorder in British Columbia. J FAS Int 2004;2:1-12.

5. Popova S, Lange S, Bekmuradov D, et al. Fetal alcohol spectrum disorder prevalence estimates in correctional systems: a systematic literature review. Can J Public Health 2011;102:336-40.

6. Watkins RE, Elliott EJ, Wilkins A, et al. Recommendations from a consensus development workshop on the diagnosis of fetal alcohol spectrum disorders in Australia. BMC Pediatr 2013;13:156-65.

7. Streissguth A, Barr H, Kogan J, et al. Primary and secondary disabilities in fetal alcohol syndrome. In: Streissguth A, Kanter J, eds. The challenge of fetal alcohol syndrome: overcoming secondary disabilities. Seattle, WA: University of Washington Press, 1997:25-39.

8. Connor PD, Sampson PD, Bookstein FL, et al. Direct and indirect effects of prenatal alcohol damage on executive function. Dev Neuropsychol 2000;18:331-54

9. Fast DK, Conry J. Fetal alcohol spectrum disorders and the criminal justice system. Dev Disabil Res Rev 2009;15:250-7.

10. Loeber R, Farrington DP. Young children who commit crime: epidemiology, developmental origins, risk factors, early interventions, and policy implications. Dev Psychopatho 2000;12:737-62.

11. Scott ES, Steinberg LD. Rethinking juvenile justice. Cambridge, MA Harvard University Press, 2009.

12. Brown J, Russell A, Wartnik AP, et al. FASD and the juvenile justice system: a need for increased awareness. J Law Enforcement 2015;4:28-39.

13. Burd L, Selfridge R, Klug M, et al. Fetal alcohol syndrome in the United States corrections system. Addict Biol 2004;9:169-76.

14. Chasnoff IJ, Wells AM, King L. Misdiagnosis and missed diagnoses in foster and adopted children with prenatal alcohol exposure. Pediatrics 2015;135:264-70.

15. Brown NN, Burd L, Grant T, et al. Prenatal alcohol exposure: an assessment strategy for the legal context. Int J Law Psychiatry 2015;42-3:144-8.

16. Fitzpatrick JP, Latimer J, Carter M, et al. Prevalence of fetal alcohol syndrome in a population-based sample of children living in remote Australia: the Lililwan Project. J Paediatr Child Health 2015:51:450-7.

17. Elliott EJ, Payne J, Morris A, et al. Fetal alcohol syndrome: a prospective national surveillance study. Arch Dis Child 2008:93:732-7.

18. Mutch RC, Watkins R, Bower C. Fetal alcohol spectrum disorders: notifications to the Western Australian Register of Developmental Anomalies. J Paediatr Child Health 2015;51:433-6.

19. Australian Institute of Health and Welfare. Juvenile detention population in Australia 2012. Juvenile Justice Series no 11 Cat no JUV 11. Canberra, ACT: Australian Institute of Health and Welfare, 2012.

20. Allely CS, Gebbia P. Studies investigating fetal alcohol spectrum disorders in the criminal justice system: a systematic PRISMA review. SOJ Psychol 2016;2:1-11.

21. Popova S, Lange S, Burd L, et al. Cost attributable to fetal alcohol spectrum disorder in the Canadian correctional system. Int J Law Psychiatry 2015;41:76-81.

22. Payne J, Elliott E, D'Antoine $\mathrm{H}$, et al. Health professionals' knowledge, practice and opinions about fetal alcohol syndrome and alcohol consumption in pregnancy. Aust N Z J Public Health 2005;29:558-64.

23. Payne JM, France KE, Henley N, et al. Paediatricians' knowledge, attitudes and practice following provision of educational resources about prevention of prenatal alcohol exposure and Fetal Alcohol Spectrum Disorder. J Paediatr Child Health 2011;47:704-10.

24. Scott IA, Glasziou PP. Improving effectiveness of clinical medicine: the need for better translation of science into practice. Med J Aust 2012;197:374-8

25. Watkins RE, Elliott EJ, Halliday J, et al. A modified Delphi study of screening for fetal alcohol spectrum disorders in Australia. BMC Pediatr 2013;13:13-24.

26. Elliott EJ, Payne J, Haan E, et al. Diagnosis of foetal alcohol syndrome and alcohol use in pregnancy: a survey of paediatricians' knowledge, attitudes and practice. J Paediatr Child Health 2006;42:698-703.

27. Peadon E, Fremantle E, Bower C, et al. International survey of diagnostic services for children with fetal alcohol spectrum disorders. BMC Pediatr 2008;8:12-20.

28. Chudley AE, Conry J, Cook JL, et al. Fetal alcohol spectrum disorder: Canadian guidelines for diagnosis. CMAJ 2005;172(5 Suppl):S1-21

29. Carmichael Olson H, Jirikowic T, Kartin D, et al. Responding to the challenge of early intervention for fetal alcohol spectrum disorders. Infants Young Child 2007;20:172-89.

30. McLachlan K, Roesch R, Viljoen JL, et al. Evaluating the psycholegal abilities of young offenders with fetal alcohol spectrum disorder. Law Hum Behav 2014;38:10-22.

31. Education and Health Standing Committee. Foetal alcohol spectrum disorder: the invisible disability. Western Australia Parliament Legislative Assembly Committees: Education and Health Standing Committee Report 15. Perth, WA: Parliament of Western Australia, 2012.

32. House of Representatives Standing Committee on Social Policy and Legal Affairs. FASD: the hidden harm, inquiry into the prevention, diagnosis and management of fetal alcohol spectrum disorders. Canberra, ACT: The Department of the House of Representatives, 2012.

33. House of Representatives Standing Committee on Aboriginal and Torres Strait Islander Affairs. Doing time-time for doing: indigenous youth in the criminal justice system. Canberra, ACT: Parliament of the Commonwealth of Australia, 2011.

34. Youth Justice Think Tank. The report and recommendations of the 2012 Youth Justice Think Tank: building a more effective youth justice system in WA. Perth, WA: Youth Affairs Council of Western Australia, Youth Legal Service and Western Australian Council of Social Services, 2013.

35. Department of Health WA. Fetal alcohol spectrum disorder model of care. Perth, WA: Health Networks Branch, Department of Health, Western Australia, 2010.

36. Office of the Inspector of Custodial Services. Report of an announced inspection of Banksia Hill Juvenile Detention Centre. Perth, WA, 2015.

37. Bush K, Kivlahan DR, McDonell MB, et al. The AUDIT Alcohol Consumption Questions (AUDIT-C): an effective brie screening test for problem drinking. Arch Intern Med 1998;158:1789-95.

38. Achenbach TM, Rescorla LA. Manual for the ASEBA School-Age Forms and Profiles. Burlington, VT: Research Center for Children, Youth, and Families, University of Vermont, 2001

39. Sparrow S, Chicchettie D, Balla D. Vineland adaptive behavior scales, parent/caregiver rating form. 2nd edn. Minneapolis, MN NCS Pearson Inc, 2005.

40. Gioia GA, Isquith PK, Guy SC, et al. Behavior Rating Inventory of Executive Function: BRIEF. Odessa, FL: Psychological Assessment Resources, 2000

41. Sparrow S, Cicchetti D, Balla D. Vineland adaptive behavior scales teacher rating form. 2nd edn. Minneapolis, MN: NCS Pearson Inc, 2006.

42. Conry J, Asante K. Youth probation officers' guide to FASD screening and referral. Maple Ridge, BC: The Asante Centre for Fetal Alcohol Syndrome, 2010.

43. Ainge D. Life skills checklist for students for identify as Indigenous. Innovations Educ Teach International 2001;39:107-16.

44. Astley SJ. Diagnostic guide for fetal alcohol spectrum disorders: the 4-digit diagnostic code. 3rd edn. Seattle, WA: University of Washington Publication Services, 2004.

45. Mutch $\mathrm{RC}$, Cherian $\mathrm{S}, \mathrm{Nemba} \mathrm{K}$, et al. Tertiary paediatric refugee health clinic in Western Australia: analysis of the first 1026 children $J$ Paediatr Child Health 2012;48:582-7.

46. Chitsabesan P, Lennox C, Theodosiou L, et al. The development of the comprehensive health assessment tool for young offenders within the secure estate. J Forens Psychiatry Psychol 2014;25: $1-25$.

47. Cohen E, Mackenzie RG, Yates GL. HEADSS, a psychosocial risk assessment instrument: implications for designing effective intervention programs for runaway youth. $J$ Adolesc Health 1991;12:539-44.

48. Wechsler D. Wechsler abbreviated scale of intelligence. 2nd edn Bloomington, MN: Pearson, 2011.

49. Wechsler D, Naglieri JA. Wechsler nonverbal scale of ability. San Antonio, TX: Harcourt Assessment, 2006.

50. Delis D, Kaplan E, Kramer J. Delis-Kaplan executive function system. San Antonio, TX: The Psychological Corporation, Harcourt Brace \& Company, 2001. 
51. Adams W, Sheslow D. Wide range assessment of memory and learning. 2nd edn. Wilmington, DE: Wide Range, 2004.

52. Wilkinson GD, Robertson GJ. Wide range achievement test. 4th edn. Lutz, FL: Psychological Assessment Resources, 2006.

53. Semel E, Wiig EH, Secord WA. Clinical Evaluation of Language Fundamentals Fourth Edition, Australian Standardised Edition (CELF-4 Australian). Sydney, NSW: Pearson Clinical and Talent Assessment, 2003

54. Gould J. Non-standard assessment practices in the evaluation of communication in Australian Aboriginal children. Clin Linguist Phon 2008;22:643-57.

55. Wagner R, Torgesen J, Rashotte C. Comprehensive Test of Phonological Processing Second Edition (CTOPP-2). Texas, TX Pro-Ed Incorporated, 2013.

56. Snow PC, Powell MB. What's the story? An exploration of narrative language abilities in male juvenile offenders. Psychol Crime Law 2005;11:239-53.

57. Semel E, Wiig EH, Secord WA. Clinical Evaluation of Language Fundamentals Fourth Edition, Screening Test Australian \& New Zealand Language Adapted Edition (CELF-4 Screener). Sydney, NSW: Pearson Clinical and Talent Assessment, 2004.

58. Beery K, Beery N. The Beery-Buktenica Development Test of Visual-Motor Integration: administration, scoring and teaching manual. 6th edn. Minneapolis, MN: Pearson, 2010.

59. Henderson AJ, Sugden DA, Barnett AL. Movement Assessment Battery for Children Second Edition (Movement ABC-2). London, UK: The Psychological Corporation, 2007.

60. Mutti MA, Sterling MD, Martin NA, et al. Quick Neurological Screening Test Manual Third Edition (QNST-3). Novato, CA Academic Therapy Publications, 2012.

61. Brown C, Dunn W. Adolescent/adult sensory profile user's manual. Bloomington, MN: Psychological Corp, 2002.

62. Pearce WM, Williams C. The cultural appropriateness and diagnostic usefulness of standardized language assessments for Indigenous Australian children. Int J Speech Lang Pathol 2013;15:429-40.

63. Watkins RE, Elliott EJ, Mutch RC, et al. Consensus diagnostic criteria for fetal alcohol spectrum disorders in Australia: a modified Delphi study. BMJ Open 2012;2:e001918.

64. Proven S, Ens C, Beaudin PG. The language profile of school-aged children with fetal alcohol spectrum disorder (FASD). Can J Speech Lang Pathol Audiol 2014;37:268-79.

65. Snow PC, Powell MB. Oral language competence in incarcerated young offenders: links with offending severity. Int J Speech Lang Pathol 2011;13:480-9.

66. Coggins TE, Timler GR, Olswang LB. A state of double jeopardy: impact of prenatal alcohol exposure and adverse environments on the social communicative abilities of school-age children with fetal alcohol spectrum disorder. Lang Speech Hear Serv Sch 2007;38:117-27.

67. Games F, Curran A, Porter S. A small-scale pilot study into language difficulties in children who offend. Educ Psychol Pract 2012;28:127-40.

68. Cahir $\mathrm{P}$. Examining culturally valid language assessments for Indigenous children. ACQ 2011;13:120-5.
69. Gould J. Language difference or language disorder: discourse sampling in speech pathology assessments for Indigenous children. In: Simpson J, Wigglesworth G, eds. Children's language and multilingualism: indigenous language use at home and school. London, UK: Continuum, 2008:194-215.

70. Malcolm IG. Issues in English language assessment of Indigenous Australians. Lang Assess Q 2011;8:190-9.

71. Department of Education and Training. ESL/ESD progress map, English as a second language, English as a second dialect: professional guidelines, listening, speaking, reading \& viewing, and writing. West Perth, WA: WestOne Services, 2009

72. Salter C. Conducting communication assessments with school aged Aboriginal Children in the Kimberley region of Australia. Aust Rev Appl Linguist 2013;36:316-34.

73. Fitzpatrick JP, Elliott EJ, Latimer J, et al. The Lililwan Project study protocol for a population-based active case ascertainment study of the prevalence of Fetal Alcohol Spectrum Disorders (FASD) in remote Australian Aboriginal communities. BMJ Open 2012;2:e000968.

74. Wiig EH, Secord WA. Clinical Evaluation of Language Fundamentals, Fifth Edition Metalinguistics (CELF-5 Metalinguistics). Sydney, NSW: Pearson Clinical and Talent Assessment, 2014.

75. Mattson SN, Roesch SC, Fagerlund A, et al. Toward a neurobehavioral profile of fetal alcohol spectrum disorders. Alcohol Clin Exp Res 2010;34:1640-50.

76. Buderer NM. Statistical methodology: I. Incorporating the prevalence of disease into the sample size calculation for sensitivity and specificity. Acad Emerg Med 1996;3:895-900.

77. Bower C, Silva D, Henderson TR, et al. Ascertainment of birth defects: the effect on completeness of adding a new source of data. J Paediatr Child Health 2000;36:574-6.

78. English D, Bower C. Alcohol consumption, pregnancy and low birthweight (letter). Lancet 1983;1:1111.

79. Peadon E, Rhys-Jones B, Bower C et al. Systematic review of interventions for children with fetal alcohol spectrum disorder J Paediatr Child Health 2007;43:A1-22.

80. O'Leary CM, Bower C. Guidelines for pregnancy: what's an acceptable risk, and how is the evidence (finally) shaping up? Drug Alcohol Rev 2012;31:170-83.

81. Elliott EJ, Bower C. FAS in Australia: fact or fiction? J Paediatr Child Health 2004;40:8-10.

82. Burd L, Selfridge RH, Klug MG, et al. Fetal alcohol syndrome in the Canadian corrections system. J FAS Int 2003;1:1-10.

83. Streissguth AP, Barr HM, Kogan J, et al. Understanding the occurrence of secondary disabilities in clients with fetal alcohol syndrome (FAS) and fetal alcohol effects (FAE), final report to the Centers for Disease Control and Prevention (CDC). Seattle: University of Washington, Fetal Alcohol \& Drug Unit, 1996.

84. Telethon Kids Institute. FASD \& Justice-Professional Development Secondary FASD \& Justice-Professional Development, 2015. http://alcoholpregnancy.telethonkids.org.au/fasd-justice/ professional-development/

85. Yin RK. Case study research: design and methods. Thousand Oaks, CA: Sage Publications, 2013. 